\title{
Endomorphisms of Clifford semigroups with injective structure homomorphisms
}

\author{
S. Worawiset* and J. Koppitz
}

\author{
Communicated by A. V. Zhuchok
}

\begin{abstract}
In the present paper, we study semigroups of endomorphisms on Clifford semigroups with injective structure homomorphisms, where the semilattice has a least element. We describe such Clifford semigroups having a regular endomorphism monoid. If the endomorphism monoid on the Clifford semigroup is completely regular then the corresponding semilattice has at most two elements. We characterize all Clifford semigroups $G_{\alpha} \cup G_{\beta}$ $(\alpha>\beta)$ with an injective structure homomorphism, where $G_{\alpha}$ has no proper subgroup, such that the endomorphism monoid is completely regular. In particular, we consider the case that the structure homomorphism is bijective.
\end{abstract}

\section{Introduction}

Inverse semigroups are a well studied class of semigroups. A completely regular inverse semigroup is called Clifford semigroup or also strong semilattice of groups. Let $Y$ be a semilattice, i.e. an idempotent semigroup with $\alpha \beta=\beta \alpha$, for all $\alpha, \beta \in Y$. The partial order relation $\geqslant$ in $Y$ is that obtained from the semilattice operation. Let $G_{\xi}$ be a group for each $\xi \in Y$ with $G_{\alpha} \cap G_{\beta}=\varnothing$, whenever $\alpha \neq \beta$. For $\xi \in Y$, we will mean by $x_{\xi}$ an element in the group $G_{\xi}$ and $e_{\xi}$ denotes the identity element in the group

*Partially supported by the Thai Visiting Scholar 2019, Khon Kaen University, Thailand.

2010 MSC: 20M10, 20M15.

Key words and phrases: Clifford semigroups, endomorphism monoid, regular. 
$G_{\xi}$. For each pair $\alpha \geqslant \beta$ of elements in $Y$, let $\varphi_{\alpha, \beta}: G_{\alpha} \rightarrow G_{\beta}$ be a group homomorphism such that $\varphi_{\alpha, \alpha}$ is the identity automorphism on $G_{\alpha}$, for all $\alpha \in Y$ and if $\alpha \geqslant \beta \geqslant \gamma$ then $\varphi_{\alpha, \gamma}=\varphi_{\alpha, \beta} \varphi_{\beta, \gamma}$. We will write mappings on the right of the objects on which they act. In $S=\bigcup_{\xi \in Y} G_{\xi}$, we take the multiplication

$$
x_{\alpha} * x_{\beta}=\left(x_{\alpha}\right) \varphi_{\alpha, \alpha \beta}\left(x_{\beta}\right) \varphi_{\beta, \alpha \beta}
$$

for $\alpha, \beta \in Y$. The semigroup $S$ is a strong semilattice $Y$ of groups $G_{\xi}$, i.e. a Clifford semigroup and each Clifford semigroup is of this form [2]. Basic facts about Clifford semigroups can be found in any introductory book on semigroup theory, for example in [4,7]. For each pair $\alpha \geqslant \beta \in Y$, we call $\varphi_{\alpha, \beta}: G_{\alpha} \rightarrow G_{\beta}$ the structure homomorphism or defining homomorphism. Although monoids of endomorphisms on Clifford semigroups seem a natural object of study, not so much has be done on this subject. Of course, each group is a Clifford semigroup. Semigroups of endomorphisms on groups are studied in $[3,8,9]$. Let $S$ and $T$ be semigroups and let $f: S \rightarrow T$ be a mapping. Then $f$ is called a semigroup homomorphism if $(x y) f=(x) f(y) f$ for all $x, y \in S$. The homomorphism $f$ is called endomorphism if $S=T$. The set of all semigroup homomorphisms (of all endomorphisms) is denoted by $\operatorname{Hom}(S, T)$ (by $\operatorname{End}(S)=\operatorname{Hom}(S, S))$. The image of a homomorphism $f \in \operatorname{Hom}(S, T)$ is denoted by $\operatorname{Im}(f)=\{(x) f: x \in S\}$. Endomorphisms on Clifford semigroups were studied for examples in $[10,11]$.

An element $x$ of a semigroup $S$ is called regular if there exists an element $y \in S$ such that $x y x=x$. In this sense, $y$ is called pseudoinverse of $x$. An element $a$ is called inverse of $x$ if $x a x=x$ and $a x a=a$. The semigroup $S$ is called regular if each element has at least one pseudoinverse or equivalently, each element has at least one inverse element. In the present paper, we use only the existence of a pseudoinverse for each element in order to verify the regularity of a semigroup. An element $x$ of a semigroup $S$ is called completely regular if there exists an element $y \in S$ such that $x y x=x$ and $x y=y x$. The semigroup $S$ is called completely regular if all its elements are completely regular. A semigroup $S$ is called idempotent or band if $x x=x$ for all $x \in S$. See for examples in $[4,7]$

A semigroup $S$ is called endo-regular if its monoid of endomorphisms is regular. The regularity of the endomorphism monoid for groups were studied by several authors (see for examples [1,5]). In particular, Theorem 1.2 in [6] gives a characterization of regular endomorphisms on groups. It appears the question when each endomorphism has a pseudoinverse, i.e, when the endomorphism monoid of a group is regular. In [6], John Meldrum 
gives a partial answer on this question. In the present paper, we want to ask this question for Clifford semigroups. It seems almost impossible to find an answer for the class of all Clifford semigroups. Therefore, we restrict ourselves to a class of Clifford semigroups, for which we have already some important information about their endomorphisms.

Let us consider a Clifford semigroup $S=\bigcup_{\xi \in Y} G_{\xi}$ and let $f \in \operatorname{End}(S)$. In [10], the authors show that the restriction of an endomorphism $f$ on $S$ to the set $E_{S}=\left\{e_{\xi}: \xi \in Y\right\}$ of the idempotent elements in $S$ induces an endomorphism on $Y$, which we denote by $f$. In fact, $(\alpha) f=\beta$, whenever $\left(e_{\alpha}\right) f=e_{\beta}$. This homomorphism is called the induced index mapping. We denote by $f_{\xi}$ the restriction of $f$ to the group $G_{\xi}$. Then the image of $f_{\xi}$ is a subgroup of $G_{(\xi) f}$, i.e. $f_{\xi} \in \operatorname{Hom}\left(G_{\xi}, G_{(\xi) f}\right)$, where $\left(e_{\xi}\right) f=e_{(\xi) f}$. We suppose that the structure homomorphisms are injective and the semilattice $Y$ has a least element $\nu$. Then an endomorphism $f$ on $S$ is already determined by the endomorphism $f_{\nu} \in \operatorname{Hom}\left(G_{\nu}, G_{(\nu) f}\right)$ [10]. We will study the endomorphism monoid of such a Clifford semigroup $S$. In particular, we consider the case that $\operatorname{End}(S)$ is regular and completely regular, respectively. Let note that the monoid $\operatorname{End}(S)$ is related to the monoid $\operatorname{End}(Y)$ of all endomorphisms on the semilattice $Y$. In fact, if $\operatorname{End}(S)$ is regular (idempotent and completely regular, respectively) then $\operatorname{End}(Y)$ is also regular (idempotent and completely regular, respectively) by Theorem 3.10 in [11]. We will use this fact subsequently without referring it. In [11], the author studied the endomorphisms on a semilattice $Y$. In particular, the author has characterized the semilattices $Y$ with idempotent and completely regular, respectively, endomorphism monoid $\operatorname{End}(Y)$. If $Y$ is a semilattice then $\operatorname{End}(Y)$ is completely regular if and only if it is idempotent if and only if $|Y| \leqslant 2$.

\section{Regular endomorphism monoid}

In this section, we consider endo-regular Clifford semigroups $S=$ $\bigcup_{\xi \in Y} G_{\xi}$ with injective structure homomorphisms $\varphi_{\alpha, \beta}$, for $\alpha>\beta \in Y$, where $Y$ is a semilattice with a least element $\nu=\bigwedge Y$. We start with three propositions which include some already known results. In [10], it is shown that any $f \in \operatorname{End}(S)$ is determined by $f_{\nu}$.

Proposition 1. [10] Let $Y$ be a semilattice which has a least element $\nu=\bigwedge Y$ and let $S=\bigcup_{\xi \in Y} G_{\xi}$ be a Clifford semigroup with injective structure homomorphisms $\varphi_{\alpha, \beta}$, for $\alpha>\beta \in Y$. If $f \in \operatorname{End}(S)$ then $f_{\xi}=\varphi_{\xi, \nu} f_{\nu} \varphi_{(\xi) \underline{f},(\nu) \underline{f}}^{-1}$, for all $\xi \in Y$. 
In particular, we have $\operatorname{Im}\left(\varphi_{\xi, \nu}\right) f_{\nu} \subseteq \operatorname{Im}\left(f_{(\xi) f,(\nu) f}\right)$. We will use this fact subsequently without mentioning it.

On the other hand, in [11], the author constructs an endomorphism on $S$ based on an endomorphism on $G_{\nu}$. This construction does not require that the structure homomorphisms are injective.

Proposition 2. [11] Let $Y$ be a semilattice which has a least element $\nu=\bigwedge Y$ and let $S=\bigcup_{\xi \in Y} G_{\xi}$ be a Clifford semigroup. For $g \in \operatorname{End}\left(G_{\nu}\right)$, we define $f: S \rightarrow S$ by $\left(x_{\xi}\right) f=\left(x_{\xi}\right) f_{\xi}$, where

$$
f_{\xi}=\varphi_{\xi, \nu} g \quad \text { for } \xi \in Y \text {. }
$$

Then $f \in \operatorname{End}(S)$.

In [10], Samman and Meldrum constructed an endomorphism on $S$ starting from a semilattice homomorphism and a family of group homomorphisms.

Proposition 3. [10] Let $Y$ be a semilattice and let $S=\bigcup_{\xi \in Y} G_{\xi}$ be a Clifford semigroup. Given an endomorphism $s \in \operatorname{End}(Y)$ and a family $\left\{f_{\xi} \in \operatorname{Hom}\left(G_{\xi}, G_{(\xi) s}\right): \xi \in Y\right\}$ satisfying

$$
\varphi_{\alpha, \beta} f_{\beta}=f_{\alpha} \varphi_{(\alpha) s,(\beta) s},
$$

for all pairs $\alpha>\beta \in Y$. Then $f: S \rightarrow S$ defined by $\left(x_{\xi}\right) f=\left(x_{\xi}\right) f_{\xi}$, for every $\xi \in Y$, is an endomorphism on $S$.

As Propositions 1 and 2 show, the group $G_{\nu}$ plays an important part for the description of the endomorphisms on $S$. Hence, we expect that $\operatorname{End}\left(G_{\nu}\right)$ is regular, i.e. $G_{\nu}$ is an endo-regular group, whenever $S$ is endoregular (independently from the kind of the structure homomorphisms). Therefore, let us consider the set $\operatorname{End}_{\nu}(S)=\{f \in \operatorname{End}(S):(\nu) f=\nu\}$. It is easy to verify that $\operatorname{End}_{\nu}(S)$ is a submonoid of $\operatorname{End}(S)$.

Lemma 4. Let $Y$ be a semilattice which has a least element $\nu=\bigwedge Y$ and let $S=\bigcup_{\xi \in Y} G_{\xi}$ be a Clifford semigroup. If each $f \in \operatorname{End}_{\nu}(S)$ is regular in $\operatorname{End}(S)$ then $\operatorname{End}\left(G_{\nu}\right)$ is regular.

Proof. Suppose that each $f \in \operatorname{End}_{\nu}(S)$ is regular. Let $g \in \operatorname{End}\left(G_{\nu}\right)$ and let $\varphi_{\alpha, \beta}: G_{\alpha} \rightarrow G_{\beta}$, for $\alpha>\beta \in Y$, be the structure homomorphisms. Then, we define a mapping $f: S \rightarrow S$ by $\left(x_{\xi}\right) f=\left(x_{\xi}\right) f_{\xi}$ with $f_{\xi}=\varphi_{\xi, \nu} g$ for $\xi \in Y$. Note that $f_{\nu}=g$. By Proposition 2, we can conclude that $f \in \operatorname{End}(S)$ and, in particular, $f \in \operatorname{End}_{\nu}(S)$. Then there is $h \in \operatorname{End}(S)$ 
with $f h f=f$. The restriction of $f$ to the group $G_{\nu}$ provides $g=f_{\nu}=$ $f_{\nu} h_{(\nu) \underline{f}} f_{(\nu) \underline{f} \underline{h}}=f_{\nu} h_{\nu} f_{(\nu) \underline{h}}=g h_{\nu} \varphi_{(\nu) \underline{h}, \nu} g$, i.e. $h_{\nu} \varphi_{(\nu) \underline{h}, \nu}: G_{\nu} \rightarrow G_{\nu}$ is a pseudoinverse of $g$.

As already mentioned, $\operatorname{End}_{\nu}(S)$ is a submonoid of $\operatorname{End}(S)$. The following proposition characterizes Clifford semigroups $S$ (of the type that we consider) such that $\operatorname{End}_{\nu}(S)$ is regular. We note that $\operatorname{End}_{\nu}(Y)=$ $\left\{f: f \in \operatorname{End}_{\nu}(S)\right\}$ is regular, whenever $\operatorname{End}_{\nu}(S)$ is regular. In fact, if $f \in \operatorname{End}_{\nu}(S)$ then there is $h \in \operatorname{End}_{\nu}(S)$ with $f h f=f$. Then it is easy to verify that $\underline{f} \underline{h} \underline{f}=\underline{f}$ and $\underline{h} \in \operatorname{End}(Y)$.

Proposition 5. Let $Y$ be a semilattice which has a least element $\nu=\bigwedge Y$ and let $S=\bigcup_{\xi \in Y} G_{\xi}$ be a Clifford semigroup with injective structure homomorphisms. Then $\operatorname{End}_{\nu}(S)$ is regular if and only if both $\operatorname{End}_{\nu}(Y)$ and $\operatorname{End}\left(G_{\nu}\right)$ are regular.

Proof. Suppose that $\operatorname{End}_{\nu}(S)$ is regular. Then $\operatorname{End}\left(G_{\nu}\right)$ is regular by Lemma 4. Further, let $f \in \operatorname{End}_{\nu}(S)$. Then there is $h \in \operatorname{End}_{\nu}(S)$ with $f h f=f$. If we restrict both mappings $f h f$ and $f$ to the set $E_{S}$, we obtain $\underline{f} \underline{h} \underline{f}=\underline{f}$. Since $\underline{h} \in \operatorname{End}_{\nu}(Y)$, we have shown that $\operatorname{End}_{\nu}(Y)$ is regular.

Suppose now that both $\operatorname{End}_{\nu}(Y)$ and $\operatorname{End}\left(G_{\nu}\right)$ are regular. Let $f \in$ $\operatorname{End}_{\nu}(S)$. Then $t:=f \in \operatorname{End}_{\nu}(Y)$ and there is $s \in \operatorname{End}_{\nu}(Y)$ with $t s t=t$. Since $(\nu) f=\nu$, we have $f_{\nu} \in \operatorname{End}\left(G_{\nu}\right)$. Since $\operatorname{End}\left(G_{\nu}\right)$ is regular, there is $g \in \operatorname{End}\left(G_{\nu}\right)$ with $f_{\nu} g f_{\nu}=f_{\nu}$. We define a mapping $h: S \rightarrow S$ by $\left(x_{\xi}\right) h=\left(x_{\xi}\right) h_{\xi}$ with $h_{\xi}=\varphi_{\xi, \nu} g \varphi_{(\xi) s, \nu}^{-1}$, for all $\xi \in Y$. Because of

$$
\begin{aligned}
h_{\alpha} \varphi_{(\alpha) s,(\beta) s} & =\varphi_{\alpha, \nu} g \varphi_{(\alpha) s, \nu}^{-1} \varphi_{(\alpha) s,(\beta) s}=\varphi_{\alpha, \nu} g\left(\varphi_{(\alpha) s,(\beta) s} \varphi_{(\beta) s, \nu}\right)^{-1} \varphi_{(\alpha) s,(\beta) s} \\
& =\varphi_{\alpha, \beta} \varphi_{\beta, \nu} g \varphi_{(\beta) s, \nu}^{-1}=\varphi_{\alpha, \beta} h_{\beta},
\end{aligned}
$$

for all pairs $\alpha>\beta \in Y$, the mapping $h$ is an endomorphism on $S$ by Proposition 3. Note that $(\nu) \underline{h}=(\nu) s=\nu$. Hence, $h \in \operatorname{End}_{\nu}(S)$. It remains to show that $h$ is a pseudoinverse of $f$, i.e. we have to show $f_{\alpha}=f_{\alpha} h_{(\alpha) t} f_{(\alpha) t s}$, for $\alpha \in Y$. Let $\alpha \in Y$. By Proposition 1, we have $f_{\alpha}=$ $\varphi_{\alpha, \nu} f_{\nu} \varphi_{(\alpha) t, \nu}^{-1}$ and $f_{(\alpha) t s}=\varphi_{(\alpha) t s, \nu} f_{\nu} \varphi_{(\alpha) t s t, \nu}^{-1}$. Moreover, $\varphi_{(\alpha) t, \nu}^{-1} \varphi_{(\alpha) t, \nu}$ and $\varphi_{(\alpha) t s, \nu}^{-1} \varphi_{(\alpha) t s, \nu}$ map identical on $\operatorname{Im}\left(\varphi_{\alpha, \nu} f_{\nu}\right)$ and $\operatorname{Im}\left(\varphi_{(\alpha) t, \nu} g\right)$, respectively. So, we get

$$
\begin{aligned}
f_{\alpha} h_{(\alpha) t} f_{(\alpha) t s} & =\varphi_{\alpha, \nu} f_{\nu} \varphi_{(\alpha) t, \nu}^{-1} \varphi_{(\alpha) t, \nu} g \varphi_{(\alpha) t s, \nu}^{-1} \varphi_{(\alpha) t s, \nu} f_{\nu} \varphi_{(\alpha) t s t, \nu}^{-1} \\
& =\varphi_{\alpha, \nu} f_{\nu} g f_{\nu} \varphi_{(\alpha) t s t, \nu}^{-1}=\varphi_{\alpha, \nu} f_{\nu} \varphi_{(\alpha) t, \nu}^{-1}=f_{\alpha} .
\end{aligned}
$$


We turn back to the monoid $\operatorname{End}(S)$. We will now show that we only need the information about $f_{\nu}$ for all $f \in \operatorname{End}(S)$ and that $\operatorname{End}\left(G_{\nu}\right)$ is a regular group in order to verify that $S$ is endo-regular.

Lemma 6. Let $Y$ be a semilattice which has a least element $\nu=\wedge Y$ and let $S=\bigcup_{\xi \in Y} G_{\xi}$ be a Clifford semigroup with injective structure homomorphisms. Then $\operatorname{End}(S)$ is regular if and only if for any $f \in \operatorname{End}(S)$ there are $t \in \operatorname{End}(Y)$ and $g \in \operatorname{End}\left(G_{\nu}\right)$ with $\underline{f t} \underline{f}=\underline{f}$ and $\operatorname{Im}\left(\varphi_{\alpha, \nu} g\right) \subseteq$ $\operatorname{Im}\left(\varphi_{(\alpha) t, \nu}\right)$, for any $\alpha \in Y$, such that

$$
f_{\nu} \varphi_{(\nu), f^{\prime}} g f_{\nu}=f_{\nu} .
$$

Proof. Suppose that $\operatorname{End}(S)$ is regular and let $f \in \operatorname{End}(S)$. Then there is $h \in \operatorname{End}(S)$ such that $f h f=f$. We put $g^{*}:=h_{\nu}$ and $t:=\underline{h} \in$ $\operatorname{End}(Y)$. Then $f h f=f$ implies sts $=s$, where $s:=f$. By Proposition 1 , we have $h_{(\nu) s}=\varphi_{(\nu) s, \nu} g^{*} \varphi_{(\nu) s t,(\nu) t}^{-1}$ and $f_{(\nu) s t}=\varphi_{(\nu) s t, \nu} f_{\nu} \varphi_{(\nu) s t s,(\nu) s}^{-1}=$ $\varphi_{(\nu) s t, \nu} f_{\nu} \varphi_{(\nu) s,(\nu) s}^{-1}=\varphi_{(\nu) s t, \nu} f_{\nu}$. The restriction of $f h f(=f)$ to $G_{\nu}$ provides $f_{\nu}=f_{\nu} h_{(\nu) s} f_{(\nu) s t}=f_{\nu} \varphi_{(\nu) s, \nu} g^{*} \varphi_{(\nu) s t,(\nu) t}^{-1} \varphi_{(\nu) s t, \nu} f_{\nu}=f_{\nu} \varphi_{(\nu) s, \nu} g^{*} \varphi_{(\nu) t, \nu} f_{\nu}$.

We have $f_{\nu} \varphi_{(\nu) s, \nu} g f_{\nu}=f_{\nu}$ and $g=g^{*} \varphi_{(\nu) t, \nu} \in \operatorname{End}\left(G_{\nu}\right)$. Let $\alpha \in Y$. From $h_{\alpha}=\varphi_{\alpha, \nu} g^{*} \varphi_{(\alpha) t,(\nu) t}^{-1}$ (by Proposition 1), we obtain $\operatorname{Im}\left(\varphi_{\alpha, \nu} g^{*}\right) \subseteq$ $\operatorname{Im}\left(\varphi_{(\alpha) t,(\nu) t}\right)$ and thus

$$
\operatorname{Im}\left(\varphi_{\alpha, \nu} g^{*}\right) \varphi_{(\nu) t, \nu} \subseteq \operatorname{Im}\left(\varphi_{(\alpha) t,(\nu) t}\right) \varphi_{(\nu) t, \nu}=\operatorname{Im}\left(\varphi_{(\alpha) t, \nu}\right) .
$$

Thus, $\operatorname{Im}\left(\varphi_{\alpha, \nu} g\right)=\operatorname{Im}\left(\varphi_{\alpha, \nu} g^{*}\right) \varphi_{(\nu) t, \nu} \subseteq \operatorname{Im}\left(\varphi_{(\alpha) t, \nu}\right)$.

Suppose now that for all $f \in \operatorname{End}(S)$, there are $t \in \operatorname{End}(Y)$ and $g \in \operatorname{End}\left(G_{\nu}\right)$ with $\underline{f t} \underline{f}=\underline{f}$ and $\operatorname{Im}\left(\varphi_{\alpha, \nu} g\right) \subseteq \operatorname{Im}\left(\varphi_{(\alpha) t, \nu}\right)$, for all $\alpha \in Y$, such that $f_{\nu} \varphi_{(\nu) \underline{f}, \nu} g \bar{f}_{\nu}=f_{\nu}^{-}$. Let $f \in \operatorname{End}(S)$. Then there exist $t \in \operatorname{End}(Y)$ such that $s t s=s$ with $s:=f$ and $g \in \operatorname{End}\left(G_{\nu}\right)$ such that $\operatorname{Im}\left(\varphi_{\alpha, \nu} g\right) \subseteq$ $\operatorname{Im}\left(\varphi_{(\alpha) t, \nu}\right)$, for all $\alpha \in Y$ with $f_{\nu} \varphi_{(\nu) s, \nu} g f_{\nu}=f_{\nu}$. Now we can define a mapping $h: S \rightarrow S$ with

$$
\left(x_{\alpha}\right) h=\left(x_{\alpha}\right) h_{\alpha},
$$

where $h_{\alpha}=\varphi_{\alpha, \nu} g \varphi_{(\alpha) t, \nu}^{-1}$, for all $\alpha \in Y$. Because of

$$
\operatorname{Im}\left(\varphi_{\alpha, \nu} g\right) \subseteq \operatorname{Im}\left(\varphi_{(\alpha) t, \nu}\right),
$$

for all $\alpha \in Y$, the mapping $h$ is well defined. We will show that

$$
\varphi_{\alpha, \beta} h_{\beta}=h_{\alpha} \varphi_{(\alpha) s,(\beta) s},
$$


for all pairs $\alpha>\beta \in Y$. We have

$$
\begin{aligned}
\varphi_{\alpha, \beta} h_{\beta} & =\varphi_{\alpha, \beta} \varphi_{\beta, \nu} g \varphi_{(\beta) t, \nu}^{-1}=\varphi_{\alpha, \nu} g \varphi_{(\beta) t, \nu}^{-1} \varphi_{(\alpha) t,(\beta) t}^{-1} \varphi_{(\alpha) t,(\beta) t} \\
& =\varphi_{\alpha, \nu} g \varphi_{(\alpha) t, \nu}^{-1} \varphi_{(\alpha) t,(\beta) t}=h_{\alpha} \varphi_{(\alpha) t,(\beta) t} .
\end{aligned}
$$

Thus, $h \in \operatorname{End}(S)$ by Proposition 3 .

Finally, we show that $f h f=f$. Let $\alpha \in Y$. Using the fact that $f_{\alpha}=$ $\varphi_{\alpha, \nu} f_{\nu} \varphi_{(\alpha) s,(\nu) s}-1$ and $f_{(\alpha) s t}=\varphi_{(\alpha) s t, \nu} f_{\nu} \varphi_{(\alpha) s t s,(\nu) s}^{-1}=\varphi_{(\alpha) s t, \nu} f_{\nu} \varphi_{(\alpha) s,(\nu) s}^{-1}$ and since $h_{(\alpha) s} \in \operatorname{Hom}\left(G_{(\alpha) s}, G_{(\alpha) s t}\right)$, we get

$$
\begin{aligned}
f_{\alpha} h_{(\alpha) s} f_{(\alpha) s t} & =\varphi_{\alpha, \nu} f_{\nu} \varphi_{(\alpha) s,(\nu) s}^{-1} \varphi_{(\alpha) s, \nu} g \varphi_{(\alpha) s t, \nu}^{-1} \varphi_{(\alpha) s t, \nu} f_{\nu} \varphi_{(\alpha) s,(\nu) s}^{-1} \\
& =\varphi_{\alpha, \nu} f_{\nu} \varphi_{(\nu) s, \nu} g f_{\nu} \varphi_{(\alpha) s,(\nu) s}^{-1}=\varphi_{\alpha, \nu} f_{\nu} \varphi_{(\alpha) s,(\nu) s}^{-1}=f_{\alpha} .
\end{aligned}
$$

This shows that $f h f=f$ and consequently, $\operatorname{End}(S)$ is regular.

Lemma 4 states that $G_{\nu}$ is necessarily an endo-regular group in case that $\operatorname{End}(S)$ is regular. If all the groups in the Clifford semigroup are pairwise isomorphic, i.e. the structure homomorphisms are bijective, then it is sufficient for $S$ to be endo-regular that $G_{\nu}$ is an endo-regular group. This is an immediate consequence of Lemma 6 .

Corollary 7. Let $Y$ be a semilattice which has a unique least element $\nu=\bigwedge Y$ and let $S=\bigcup_{\xi \in Y} G_{\xi}$ be a Clifford semigroup with bijective structure homomorphisms. Then $\operatorname{End}(S)$ is regular if and only if both $\operatorname{End}(Y)$ and $\operatorname{End}\left(G_{\nu}\right)$ are regular.

Proof. Suppose that $\operatorname{End}(S)$ is regular. Then $\operatorname{End}\left(G_{\nu}\right)$ is regular by Lemma 4. Note that $\operatorname{End}(Y)$ is regular, whenever $\operatorname{End}(S)$ is regular.

Conversely, suppose that both $\operatorname{End}\left(G_{\nu}\right)$ and $\operatorname{End}(Y)$ are regular. Let $f \in \operatorname{End}(S)$. Then $s:=f \in \operatorname{End}(Y)$ and there is $t \in \operatorname{End}(Y)$ with $s t s=s$. Consider the endomorphism $f_{\nu} \varphi_{(\nu) s, \nu} \in \operatorname{End}\left(G_{\nu}\right)$. Since $\operatorname{End}\left(G_{\nu}\right)$ is regular, there is $g \in \operatorname{End}\left(G_{\nu}\right)$ with $\left(f_{\nu} \varphi_{(\nu) s, \nu}\right) g\left(f_{\nu} \varphi_{(\nu) s, \nu}\right)=f_{\nu} \varphi_{(\nu) s, \nu}$. Now, we multiply this equation with $\varphi_{(\nu) s, \nu}^{-1}$ from the right hand side. Since $\varphi_{(\nu) s, \nu} \varphi_{(\nu) s, \nu}^{-1}$ is the identity mapping on $G_{(\nu) s}$, we obtain $f_{\nu} \varphi_{(\nu) s, \nu} g f_{\nu}=f_{\nu}$. Moreover, for all $\alpha \in Y$, we have $\operatorname{Im}\left(\varphi_{\alpha, \nu} g\right) \subseteq G_{\nu}=\operatorname{Im}\left(\varphi_{(\alpha) t, \nu}\right)$ since $\varphi_{(\alpha) t, \nu}$ is a bijection. Then by Lemma 6 , we can conclude that $\operatorname{End}(S)$ is regular.

Note that any idempotent endomorphism on a semigroup is regular. So, all Clifford semigroups with idempotent endomorphism monoid have a 
regular endomorphism monoid. In the last part of this section, we will characterize all Clifford semigroups with injective structure homomorphisms having an idempotent endomorphism monoid. We recall that $|Y| \leqslant 2$ in this case [11]. If $|Y|=1$ then $S=G_{\nu}$. So, we have a group with idempotent endomorphism monoid. We focus us to the case $|Y|=2$.

Lemma 8. Let $Y=\{\alpha>\beta\}$ be a two-element chain and let $S=G_{\alpha} \cup G_{\beta}$ be a Clifford semigroup with an injective structure homomorphism $\varphi_{\alpha, \beta}$. If $\operatorname{End}(S)$ is a band then $\operatorname{End}\left(G_{\beta}\right)$ is a band.

Proof. Suppose that $\operatorname{End}(S)$ is a band and let $g \in \operatorname{End}\left(G_{\beta}\right)$. We define a mapping $f: S \rightarrow S$ by $\left(x_{\xi}\right) f=\left(x_{\xi}\right) f_{\xi}$, for $\xi \in\{\alpha, \beta\}$, with $f_{\alpha}=\varphi_{\alpha, \beta} g$ and $f_{\beta}=g$. Then $f \in \operatorname{End}(S)$ by Proposition 2 . Note that $(\beta) f=\beta$. We observe that the restriction of $f f=f$ to $G_{\beta}$ provides $g=f_{\beta}=\bar{f}_{\beta} f_{\beta}=g g$, i.e. $g$ is idempotent. Consequently, $\operatorname{End}\left(G_{\beta}\right)$ is a band.

We can show that the idempotency of both monoids $\operatorname{End}(Y)$ and $\operatorname{End}\left(G_{\beta}\right)$ is sufficient for the idempotency of $\operatorname{End}(S)$.

Proposition 9. Let $Y=\{\alpha>\beta\}$ be a two-element chain and let $S=$ $G_{\alpha} \cup G_{\beta}$ be a Clifford semigroup with an injective structure homomorphism $\varphi_{\alpha, \beta}$. Then $\operatorname{End}(S)$ is a band if and only if $\operatorname{End}\left(G_{\beta}\right)$ is a band.

Proof. If $\operatorname{End}(S)$ is a band then $\operatorname{End}\left(G_{\beta}\right)$ is a band by Lemma 8.

Suppose that $\operatorname{End}\left(G_{\beta}\right)$ is a band. Let $f \in \operatorname{End}(S)$. Then $s:=\underline{f} \in$ $\operatorname{End}(Y)$. We note that $s s=s$ by Proposition 1 , we have that $f_{\gamma}^{-}=$ $\varphi_{\gamma, \beta} f_{\beta} \varphi_{(\gamma) s,(\beta) s}^{-1}$ for $\gamma \in\{\alpha, \beta\}$. Let $\gamma \in\{\alpha, \beta\}$. Then

$$
\begin{aligned}
f_{\gamma} f_{(\gamma) s} & =\varphi_{\gamma, \beta} f_{\beta} \varphi_{(\gamma) s,(\beta) s}^{-1} \varphi_{(\gamma) s, \beta} f_{\beta} \varphi_{(\gamma) s s,(\beta) s}^{-1} \\
& =\varphi_{\gamma, \beta}\left(f_{\beta} \varphi_{(\beta) s, \beta}\right)\left(f_{\beta} \varphi_{(\beta) s, \beta}\right) \varphi_{(\beta) s, \beta}^{-1} \varphi_{(\gamma) s,(\beta) s}^{-1} \\
& =\varphi_{\gamma, \beta}\left(f_{\beta} \varphi_{(\beta) s, \beta}\right) \varphi_{(\beta) s, \beta}^{-1} \varphi_{(\gamma) s,(\beta) s}^{-1}=\varphi_{\gamma, \beta} f_{\beta} \varphi_{(\gamma) s,(\beta) s}^{-1}=f_{\gamma}
\end{aligned}
$$

since $f_{\beta} \varphi_{(\beta) s, \beta}$ belongs to the idempotent monoid $\operatorname{End}\left(G_{\beta}\right)$, i.e.

$\left(f_{\beta} \varphi_{(\beta) s, \beta}\right)\left(f_{\beta} \varphi_{(\beta) s, \beta}\right)=\left(f_{\beta} \varphi_{(\beta) s, \beta}\right)$. So, we have shown that $f f=f$ and consequently, $\operatorname{End}(S)$ is a band.

\section{Completely regular endomorphism monoid}

In this section, we study Clifford semigroups with completely regular endomorphism monoid. In fact, if $S=\bigcup_{\xi \in Y} G_{\xi}$ is a Clifford semigroup with a completely regular endomorphism monoid then the semilattice $Y$ 
has at most two elements [11]. If $Y$ is the trivial semilattice consisting of a singleton element then $S$ is a group and we have only to consider groups with completely regular endomorphism monoid. So, it remains the case that $Y$ consists of two elements, i.e. $Y=\{\alpha>\beta\}$ is a two-element chain. If $Y=\{\alpha>\beta\}$ is a two element chain then $\operatorname{End}(Y)=\left\{i d_{Y}, s_{\alpha}, s_{\beta}\right\}$, where $i d_{Y}$ is the identity mapping on $Y$ and $s_{\alpha}$ (and $s_{\beta}$ ) is the constant mapping with the image $\alpha$ (and $\beta$, respectively). So, each element in $\operatorname{End}(Y)$ is idempotent. In particular, $\operatorname{End}(Y)$ is completely regular. In particular, we will consider the case that the group $G_{\alpha}$ does not have nontrivial normal subgroups. The characterization of all Clifford semigroups with completely regular endomorphism monoid is still an open problem.

Lemma 10. Let $Y=\{\alpha>\beta\}$ be a two-element chain and let $S=G_{\alpha} \cup G_{\beta}$ be a Clifford semigroup with an injective structure homomorphism $\varphi_{\alpha, \beta}$. If $\operatorname{End}(S)$ is completely regular then $\operatorname{End}\left(G_{\beta}\right)$ is completely regular.

Proof. Suppose that $\operatorname{End}(S)$ is completely regular and let $f \in \operatorname{End}\left(G_{\beta}\right)$. Then by Proposition 2, the following mapping $h: S \rightarrow S$ is an endomorphism on $S$. Let $\left(x_{\xi}\right) h=\left(x_{\xi}\right) h_{\xi}$, for $\xi \in\{\alpha, \beta\}$ with

$$
h_{\alpha}=\varphi_{\alpha, \beta} f \quad \text { and } \quad h_{\beta}=f .
$$

Since $\operatorname{End}(S)$ is completely regular, there is $g \in \operatorname{End}(S)$ with $h g h=h$ and $g h=h g$. Assume that $(\beta) g=\alpha$. Then from $g h=h g$, it follows $g_{\beta} h_{\alpha}=h_{\beta} g_{\beta}$, where $\left(x_{\beta}\right) g_{\beta} h_{\alpha} \in \bar{G}_{\beta}$ and $\left(x_{\beta}\right) h_{\beta} g_{\beta} \in G_{\alpha}$, a contradiction. Therefore, we have only to consider the case that $(\beta) g=\beta$, i.e. $g_{\beta} \in$ $\operatorname{End}\left(G_{\beta}\right)$. Now, $h g h=h$ and $g h=h g$ implies $f g_{\beta} f=\bar{f}$ and $g_{\beta} f=f g_{\beta}$. Hence, $f$ is a completely regular element in $\operatorname{End}\left(G_{\beta}\right)$. Therefore, $\operatorname{End}\left(G_{\beta}\right)$ is completely regular.

Proposition 11. Let $Y=\{\alpha>\beta\}$ be a two-element chain and let $S=G_{\alpha} \cup G_{\beta}$ be a Clifford semigroup such that $G_{\alpha}$ does not have nontrivial normal subgroups and $\operatorname{End}\left(G_{\beta}\right)$ is completely regular. Then $\operatorname{End}(S)$ is completely regular.

Proof. Let $f \in \operatorname{End}(S)$ and let $t:=f$.

Case 1: Suppose that $(\beta) t=\beta$. Then $f_{\beta} \in \operatorname{End}\left(G_{\beta}\right)$. Since $\operatorname{End}\left(G_{\beta}\right)$ is completely regular, there is $g \in \operatorname{End}\left(G_{\beta}\right)$ with $f_{\beta} g f_{\beta}=f_{\beta}$ and $g f_{\beta}=f_{\beta} g$. Because $G_{\alpha}$ does not have nontrivial normal subgroups, we can conclude that $f_{\alpha}$ is injective or $f_{\alpha}$ is the constant mapping $c_{(\alpha) t}^{\alpha}: G_{\alpha} \rightarrow\left\{e_{(\alpha) t}\right\}$.

Case 1.1: Suppose that $f_{\alpha}$ is injective with $f_{\alpha}=\varphi_{\alpha, \beta} f_{\beta} \varphi_{(\alpha) t, \beta}^{-1}$. If $(\alpha) t=\beta$ then $\operatorname{Im}\left(\varphi_{\alpha, \beta} g\right) \subseteq G_{\beta}=\operatorname{Im}\left(\varphi_{\beta, \beta}\right)=\operatorname{Im}\left(\varphi_{(\alpha) t, \beta}\right)$. Suppose 
that $(\alpha) t=\alpha$ and let $x \in \operatorname{Im}\left(\varphi_{\alpha, \beta} g\right)$. Then $f_{\beta}$ restricted to $\operatorname{Im}\left(\varphi_{\alpha, \beta}\right)$ is a bijection on $\operatorname{Im}\left(\varphi_{\alpha, \beta}\right)$ and $\varphi_{\alpha, \beta}$ is injective. Since $f_{\beta}$ restricted to $\operatorname{Im}\left(\varphi_{\alpha, \beta}\right)$ is a bijection on $\operatorname{Im}\left(\varphi_{\alpha, \beta}\right)$, there is $\bar{x} \in \operatorname{Im}\left(\varphi_{\alpha, \beta}\right)$ such that $x=$ $\bar{x} f_{\beta} f_{\beta} g=\bar{x} f_{\beta} g f_{\beta}=\bar{x} f_{\beta} \in \operatorname{Im}\left(\varphi_{\alpha, \beta}\right)$. This $\operatorname{shows} \operatorname{Im}\left(\varphi_{\alpha, \beta} g\right) \subseteq \operatorname{Im}\left(\varphi_{\alpha, \beta}\right)$. We define a mapping $h: S \rightarrow S$ by $\left(x_{\xi}\right) h=\left(x_{\xi}\right) h_{\xi}$ for any $\xi \in Y$, where $h_{\xi}=\varphi_{\xi, \beta} g \varphi_{(\xi) t, \beta}^{-1}$, for any $\xi \in Y$. Since $\operatorname{Im}\left(\varphi_{\alpha, \beta} g\right) \subseteq \operatorname{Im}\left(\varphi_{\alpha, \beta}\right)$ and $\operatorname{Im}\left(\varphi_{\beta, \beta} g\right) \subseteq G_{\beta}=\operatorname{Im}\left(\varphi_{\beta, \beta}\right)=\operatorname{Im}\left(\varphi_{(\beta) t, \beta}\right)$, the mapping $h$ is well defined. Note that $g=h_{\beta}$. Moreover, $h_{\alpha} \varphi_{(\alpha) t, \beta}=\varphi_{\alpha, \beta} g \varphi_{(\alpha) t, \beta}^{-1} \varphi_{(\alpha) t, \beta}=\varphi_{\alpha, \beta} g=$ $\varphi_{\alpha, \beta} h_{\beta}$ and $h$ is an endomorphism by Proposition 3. Now we need to show that $h$ is a pseudoinverse of $f$. Note that $f_{\beta}=f_{\beta} g f_{\beta}=f_{\beta} h_{\beta} f_{\beta}$. Hence, we have still to show that $f_{\alpha}=f_{\alpha} h_{(\alpha) t} f_{(\alpha) t}$. By Proposition 1, we have $f_{\alpha}=\varphi_{\alpha, \beta} f_{\beta} \varphi_{(\alpha) t, \beta}^{-1}$ and $f_{(\alpha) t}=\varphi_{(\alpha) t, \beta} f_{\beta} \varphi_{(\alpha) t, \beta}^{-1}$. So, we get

$$
\begin{aligned}
f_{\alpha} h_{(\alpha) t} f_{(\alpha) t} & =\varphi_{\alpha, \beta} f_{\beta} \varphi_{(\alpha) t, \beta}^{-1} \varphi_{(\alpha) t, \beta} g \varphi_{(\alpha) t, \beta}^{-1} \varphi_{(\alpha) t, \beta} f_{\beta} \varphi_{(\alpha) t, \beta}^{-1} \\
& =\varphi_{\alpha, \beta} f_{\beta} g f_{\beta} \varphi_{(\alpha) t, \beta}^{-1}=\varphi_{\alpha, \beta} f_{\beta} \varphi_{(\alpha) t, \beta}^{-1}=f_{\alpha} .
\end{aligned}
$$

Moreover, we have

$$
\begin{aligned}
h_{\alpha} f_{(\alpha) t} & =\varphi_{\alpha, \beta} g \varphi_{(\alpha) t, \beta}^{-1} \varphi_{(\alpha) t, \beta} f_{\beta} \varphi_{(\alpha) t, \beta}^{-1} \\
& =\varphi_{\alpha, \beta} g f_{\beta} \varphi_{(\alpha) t, \beta}^{-1}=\varphi_{\alpha, \beta} f_{\beta} g \varphi_{(\alpha) t, \beta}^{-1} \\
& =\varphi_{\alpha, \beta} f_{\beta} \varphi_{(\alpha) t, \beta}^{-1} \varphi_{(\alpha) t, \beta} g \varphi_{(\alpha) t, \beta}^{-1}=f_{\alpha} h_{(\alpha) t} .
\end{aligned}
$$

Case 1.2: Suppose now that $f_{\alpha}$ is a constant mapping $c_{(\alpha) t}^{\alpha}$. We define a mapping $h: S \rightarrow S$ by $\left(x_{\xi}\right) h=\left(x_{\xi}\right) h_{\xi}$ for any $\xi \in Y$, where $h_{\alpha}=c_{(\alpha) t}^{\alpha}$ and $h_{\beta}=f_{\beta} g g$. First, we will verify that $\varphi_{\alpha, \beta} f_{\beta}$ is a constant mapping. For this let $x_{\alpha}, \widehat{x}_{\alpha} \in G_{\alpha}$ and $x_{\beta}:=\left(x_{\alpha}\right) \varphi_{\alpha, \beta}, \widehat{x}_{\beta}:=\left(\widehat{x}_{\alpha}\right) \varphi_{\alpha, \beta}$. Then there is $a \in G_{\alpha}$ such that $\widehat{x}_{\beta}=\left(x_{\beta}\right)\left((a) \varphi_{\alpha, \beta}\right)$ in the $\operatorname{subgroup} \operatorname{Im}\left(\varphi_{\alpha, \beta}\right)$ of $G_{\beta}$. We have

$$
\begin{aligned}
\left(\widehat{x}_{\beta}\right) f & =\left(x_{\beta}\left((a) \varphi_{\alpha, \beta}\right)\right) f=\left(\left(x_{\beta}\right) \varphi_{\beta, \beta}(a) \varphi_{\alpha, \beta}\right) f=\left(x_{\beta} * a\right) f \\
& =\left(x_{\beta}\right) f *(a) f=\left(x_{\beta}\right) f \varphi_{\beta, \beta}((a) f) \varphi_{(\alpha) t, \beta} \\
& =\left(x_{\beta}\right) f\left(e_{(\alpha) t}\right) \varphi_{(\alpha) t, \beta}=\left(x_{\beta}\right) f e_{\beta}=\left(x_{\beta}\right) f .
\end{aligned}
$$

where $*$ is the operation on $S$. This provides $\left(\widehat{x}_{\alpha}\right) \varphi_{\alpha, \beta} f_{\beta}=\left(x_{\alpha}\right) \varphi_{\alpha, \beta} f_{\beta}$. Therefore, $\varphi_{\alpha, \beta} f_{\beta}$ is a constant mapping $c_{\beta}^{\alpha}: G_{\alpha} \rightarrow\left\{e_{\beta}\right\}$ and we can calculate that $\varphi_{\alpha, \beta} h_{\beta}=h_{\alpha} \varphi_{(\alpha) t, \beta}=c_{\beta}^{\alpha}$. Thus, $h$ is an endomorphism by Proposition 3. Further, we can show that $f h f=f$ as well as $f h=h f$. In fact, we have $f_{\beta} h_{\beta} f_{\beta}=f_{\beta} f_{\beta} g g f_{\beta}=f_{\beta} g f_{\beta} g f_{\beta}=f_{\beta}$ and $f_{\beta} h_{\beta}=f_{\beta} f_{\beta} g g=$ $f_{\beta} g g f_{\beta}=h_{\beta} f_{\beta}$. Further, it is easy to verify that $f_{\alpha} h_{(\alpha) t} f_{(\alpha) t}=f_{\alpha} h_{(\alpha) t}=$ $h_{\alpha} f_{(\alpha) t}=c_{(\alpha) t}^{\alpha}$ 
Case 2: Suppose now that $(\beta) t=\alpha$. Then $(\alpha) t=\alpha$. Since $G_{\alpha}$ does not have nontrivial normal subgroups, we conclude that $f_{\beta}$ is onto $G_{\alpha}$ or $f_{\beta}$ is the constant mapping $c_{\alpha}^{\beta}: G_{\beta} \rightarrow\left\{e_{\alpha}\right\}$. If $f_{\beta}=c_{\alpha}^{\beta}$ then $f_{\alpha}=\varphi_{\alpha, \beta} f_{\beta} \varphi_{(\alpha) t,(\beta) t}^{-1}=\varphi_{\alpha, \beta} c_{\alpha}^{\beta} \varphi_{\alpha, \alpha}^{-1}=\varphi_{\alpha, \beta} c_{\alpha}^{\beta}=c_{\alpha}^{\alpha}: G_{\alpha} \rightarrow\left\{e_{\alpha}\right\}$. This shows that $f$ is the constant mapping $c_{\alpha}^{S}: S \rightarrow\left\{e_{\alpha}\right\}$. Thus, $f$ is idempotent, i.e. $f$ is completely regular. Finally, suppose that $f_{\beta}$ is onto $G_{\alpha}$. Note that $f_{\beta} \varphi_{\alpha, \beta} \in \operatorname{End}\left(G_{\beta}\right)$. Since $\operatorname{End}\left(G_{\beta}\right)$ is completely regular, there is $u \in \operatorname{End}\left(G_{\beta}\right)$ with $\left(f_{\beta} \varphi_{\alpha, \beta}\right) u\left(f_{\beta} \varphi_{\alpha, \beta}\right)=f_{\beta} \varphi_{\alpha, \beta}$ and $\left(f_{\beta} \varphi_{\alpha, \beta}\right) u=u\left(f_{\beta} \varphi_{\alpha, \beta}\right)$. This implies $f_{\beta} \varphi_{\alpha, \beta} u f_{\beta}=f_{\beta}$ since $\varphi_{\alpha, \beta}$ is injective because $G_{\alpha}$ does not have nontrivial normal subgroups. First, we will show that $\operatorname{Im}\left(\varphi_{\alpha, \beta} u\right) \subseteq \operatorname{Im}\left(\varphi_{\alpha, \beta}\right)$. For this let $x \in \operatorname{Im}\left(\varphi_{\alpha, \beta} u\right)$. Since $f_{\beta}$ is onto $G_{\alpha}$, there is $\bar{x} \in G_{\beta}$ such that $x=\bar{x} f_{\beta} \varphi_{\alpha, \beta} u=\bar{x} u f_{\beta} \varphi_{\alpha, \beta} \in \operatorname{Im}\left(\varphi_{\alpha, \beta}\right)$. Then, we define a mapping $h: S \rightarrow S$ by $\left(x_{\xi}\right) h=\left(x_{\xi}\right) h_{\xi}$ for any $\xi \in Y$, where $h_{\alpha}=\varphi_{\alpha, \beta} u f_{\beta} \varphi_{\alpha, \beta} u \varphi_{\alpha, \beta}^{-1}$ and $h_{\beta}=u f_{\beta} \varphi_{\alpha, \beta} u \varphi_{\alpha, \beta}^{-1}$. Because of $\operatorname{Im}\left(\varphi_{\alpha, \beta} u\right) \subseteq \operatorname{Im}\left(\varphi_{\alpha, \beta}\right)$, the mapping $h$ is well defined. Further, we have $h_{\alpha} \varphi_{(\alpha) t,(\beta) t}=h_{\alpha} \varphi_{\alpha, \alpha}=h_{\alpha}=\varphi_{\alpha, \beta} u f_{\beta} \varphi_{\alpha, \beta} u \varphi_{\alpha, \beta}^{-1}=\varphi_{\alpha, \beta} h_{\beta}$. Thus, $h \in \operatorname{End}(S)$ by Proposition 3. We show now that $h$ is a pseudoinverse of $f$. Note that $f_{\alpha}=\varphi_{\alpha, \beta} f_{\beta} \varphi_{(\alpha) t,(\beta) t}=\varphi_{\alpha, \beta} f_{\beta} \varphi_{\alpha, \alpha}=\varphi_{\alpha, \beta} f_{\beta}$. We have

$$
\begin{aligned}
f_{\beta} h_{\alpha} f_{\alpha} & =f_{\beta} \varphi_{\alpha, \beta} u f_{\beta} \varphi_{\alpha, \beta} u \varphi_{\alpha, \beta}^{-1} \varphi_{\alpha, \beta} f_{\beta}=f_{\beta} \varphi_{\alpha, \beta} u f_{\beta} \varphi_{\alpha, \beta} \varphi_{\alpha, \beta}^{-1} \\
& =f_{\beta} \varphi_{\alpha, \beta} \varphi_{\alpha, \beta}^{-1}=f_{\beta}, \\
f_{\alpha} h_{\alpha} f_{\alpha} & =\varphi_{\alpha, \beta} f_{\beta} \varphi_{\alpha, \beta} u f_{\beta} \varphi_{\alpha, \beta} u \varphi_{\alpha, \beta}^{-1} \varphi_{\alpha, \beta} f_{\beta}=\varphi_{\alpha, \beta} f_{\beta} \varphi_{\alpha, \beta} u f_{\beta} \varphi_{\alpha, \beta} \varphi_{\alpha, \beta}^{-1} \\
& =\varphi_{\alpha, \beta} f_{\beta} \varphi_{\alpha, \beta} \varphi_{\alpha, \beta}^{-1}=\varphi_{\alpha, \beta} f_{\beta}=f_{\alpha},
\end{aligned}
$$

and

$$
\begin{aligned}
h_{\beta} f_{\alpha} & =u f_{\beta} \varphi_{\alpha, \beta} u \varphi_{\alpha, \beta}^{-1} \varphi_{\alpha, \beta} f_{\beta}=u f_{\beta} \varphi_{\alpha, \beta} u f_{\beta} \varphi_{\alpha, \beta} \varphi_{\alpha, \beta}^{-1} \\
& =f_{\beta} \varphi_{\alpha, \beta} u f_{\beta} \varphi_{\alpha, \beta} u \varphi_{\alpha, \beta}^{-1}=f_{\beta} h_{\alpha}
\end{aligned}
$$

as well as $f_{\alpha} h_{\alpha}=\varphi_{\alpha, \beta} f_{\beta} h_{\alpha}=\varphi_{\alpha, \beta} h_{\beta} f_{\alpha}=h_{\alpha} f_{\alpha}$. Consequently, $f$ is a completely regular element of $\operatorname{End}(S)$.

So, we can state the main result of this section concerning Clifford semigroups with completely regular endomorphism monoid.

Theorem 12. Let $Y=\{\alpha>\beta\}$ be a two-element chain and let $S=$ $G_{\alpha} \cup G_{\beta}$ such that $G_{\alpha}$ does not have nontrivial normal subgroups. Then $\operatorname{End}(S)$ is completely regular if and only if $\operatorname{End}\left(G_{\beta}\right)$ is completely regular. 
Proof. Suppose that $\operatorname{End}(S)$ is completely regular. The fact that $\operatorname{End}\left(G_{\beta}\right)$ is completely regular follows from Lemma 10 . The converse direction is given by Proposition 11.

Now, we drop the condition that $G_{\alpha}$ has no proper normal subgroups. We consider Clifford semigroups $S=G_{\alpha} \cup G_{\beta}(\alpha>\beta)$ such that $G_{\beta}$ is the direct product of $G_{\alpha}$ and a group. Note that such Clifford semigroups can be constructed whenever $G_{\beta}$ is an abelian group by the fundamental theorem of abelian groups.

Proposition 13. Let $Y=\{\alpha>\beta\}$ be a two-element chain and let $S=$ $G_{\alpha} \cup G_{\beta}$ be a Clifford semigroup with an injective structure homomorphism $\varphi_{\alpha, \beta}$, where $G_{\beta}$ is the direct product of the group $G_{\alpha}$ and a group with the identity element e such that $\left(x_{\alpha}\right) \varphi_{\alpha, \beta}=\left(x_{\alpha}, e\right)$. If $\operatorname{End}(S)$ is completely regular then $\operatorname{End}\left(G_{\alpha}\right)$ is completely regular.

Proof. Suppose that $\operatorname{End}(S)$ is completely regular. Then $\operatorname{End}\left(G_{\beta}\right)$ is completely regular by Lemma 10 . We put $A:=\operatorname{Im}\left(\varphi_{\alpha, \beta}\right)=\left\{\left(x_{\alpha}, e\right)\right.$ : $x_{\alpha} \in G_{\alpha}$. Clearly, $A$ is isomorphic to $G_{\alpha}$. We will show that $\operatorname{End}(A)$ is completely regular. For this let $f \in \operatorname{End}(A)$. We define a mapping $h: G_{\beta} \rightarrow G_{\beta}$ by

$$
\left(x_{\alpha}, x\right) h=\left(x_{\alpha}, e\right) f \cdot\left(e_{\alpha}, x\right) \text { for all }\left(x_{\alpha}, x\right) \in G_{\beta},
$$

where - is the multiplication on $G_{\beta}$. We have to show that $h$ is an endomorphism on $G_{\beta}$. It is easy to verify that

$$
\left(x_{\alpha}, e\right) \cdot\left(e_{\alpha}, x\right)=\left(e_{\alpha}, x\right) \cdot\left(x_{\alpha}, e\right) .
$$

Let $\left(x_{\alpha}, x\right),\left(\widetilde{x}_{\alpha}, \widetilde{x}\right) \in G_{\beta}$. Then we obtain

$$
\begin{aligned}
\left(\left(x_{\alpha}, x\right) \cdot\left(\widetilde{x}_{\alpha}, \widetilde{x}\right)\right) h & =\left(\left(x_{\alpha} \widetilde{x}_{\alpha}, x \widetilde{x}\right)\right) h=\left(x_{\alpha} \widetilde{x}_{\alpha}, e\right) f \cdot\left(e_{\alpha}, x \widetilde{x}\right) \\
& =\left(x_{\alpha}, e\right) f \cdot\left(\widetilde{x}_{\alpha}, e\right) f \cdot\left(e_{\alpha}, x\right) \cdot\left(e_{\alpha}, \widetilde{x}\right) \\
& =\left(x_{\alpha}, e\right) f \cdot\left(e_{\alpha}, x\right) \cdot\left(\widetilde{x}_{\alpha}, e\right) f \cdot\left(e_{\alpha}, \widetilde{x}\right) \\
& =\left(x_{\alpha}, x\right) f \cdot\left(\widetilde{x}_{\alpha}, \widetilde{x}\right) f .
\end{aligned}
$$

This shows that $h \in \operatorname{End}\left(G_{\beta}\right)$. Thus, there is $g \in \operatorname{End}\left(G_{\beta}\right)$ with $h g h=h$ and $h g=g h$ since $\operatorname{End}\left(G_{\beta}\right)$ is completely regular. We show now that the image of $g$ restricted to $A$ belongs to $A$. For this, let $\left(x_{\alpha}, e\right) \in A$. Assume that $\left(x_{\alpha}, e\right) g \notin A$. Then by $h g=g h$ and by the definition of $h$, we obtain that $\left(x_{\alpha}, e\right) h g h=\left(x_{\alpha}, e\right) g h h \notin A$. On the other hand, we have $\left(x_{\alpha}, e\right) h=\left(x_{\alpha}, e\right) f \in A$. This contradicts to $h g h=h$. Consequently, 
the image of $g$ restricted to $A$ is a subset of $A$. Note that $\varphi_{\alpha, \beta}^{-1} \varphi_{\alpha, \beta}$ is the identity mapping on $A$. Then $\delta \varphi_{\alpha, \beta}^{-1} \varphi_{\alpha, \beta}=\delta$ for any mapping $\delta$ with $\operatorname{Im}(\delta) \subseteq A$ and $\varphi_{\alpha, \beta}^{-1} \varphi_{\alpha, \beta} h=f$. Moreover, the mapping $p: A \rightarrow A$ defined by

$$
p=\varphi_{\alpha, \beta}^{-1} \varphi_{\alpha, \beta} g
$$

is an endomorphism on $A$. Thus, we obtain $f p f=f \varphi_{\alpha, \beta}^{-1} \varphi_{\alpha, \beta} g f=f g f=$ $\varphi_{\alpha, \beta}^{-1} \varphi_{\alpha, \beta} h g \varphi_{\alpha, \beta}^{-1} \varphi_{\alpha, \beta} h=\varphi_{\alpha, \beta}^{-1} \varphi_{\alpha, \beta} h g h=\varphi_{\alpha, \beta}^{-1} \varphi_{\alpha, \beta} h=f$ as well as $f p=$ $f \varphi_{\alpha, \beta}^{-1} \varphi_{\alpha, \beta} g=f g=\varphi_{\alpha, \beta}^{-1} \varphi_{\alpha, \beta} h g=\varphi_{\alpha, \beta}^{-1} \varphi_{\alpha, \beta} g h=p h=p \varphi_{\alpha, \beta}^{-1} \varphi_{\alpha, \beta} h=p f$. This shows that $f$ is a completely regular element in $\operatorname{End}(A)$. So, the proof is done.

We finish this section with the case that the structure homomorphism $\varphi_{\alpha, \beta}$ is bijective. Such Clifford semigroups with completely regular endomorphism monoid can be characterized by the fact that $\operatorname{End}\left(G_{\beta}\right)$ is completely regular (and thus, $\operatorname{End}\left(G_{\alpha}\right)$ is it also). Here, we can drop any restriction to the groups $G_{\alpha}$ and $G_{\beta}$.

Theorem 14. Let $Y=\{\alpha>\beta\}$ be a two-element chain and let $S=G_{\alpha} \cup$ $G_{\beta}$ be a Clifford semigroup with a bijective structure homomorphism $\varphi_{\alpha, \beta}$. Then $\operatorname{End}(S)$ is completely regular if and only if $\operatorname{End}\left(G_{\beta}\right)$ is completely regular.

Proof. Suppose that $\operatorname{End}(S)$ is completely regular. Then Lemma 10 shows that $\operatorname{End}\left(G_{\beta}\right)$ is completely regular.

Suppose now that $\operatorname{End}\left(G_{\beta}\right)$ is completely regular. Then $\operatorname{End}\left(G_{\alpha}\right)$ is also completely regular since $G_{\alpha}$ is isomorphic to $G_{\beta}$. Let $f \in \operatorname{End}(S)$ with $t:=f$. Then it is obvious that $(\alpha) t=\alpha$ or $(\beta) t=\beta$. We consider the case that $(\alpha) t=\alpha$. If $(\beta) t=\beta$ then the proof is similar. From $(\alpha) t=\alpha$, it follows $f_{\alpha} \in \operatorname{End}\left(G_{\alpha}\right)$. Since $\operatorname{End}\left(G_{\alpha}\right)$ is completely regular, there is $g \in \operatorname{End}\left(G_{\alpha}\right)$ with $f_{\alpha} g f_{\alpha}=f_{\alpha}$ and $f_{\alpha} g=g f_{\alpha}$. We define a mapping $h: S \rightarrow S$ by $\left(x_{\xi}\right) h=\left(x_{\xi}\right) h_{\xi}$ for $\xi \in\{\alpha, \beta\}$ with

$$
h_{\alpha}=g \quad \text { and } \quad h_{\beta}=\varphi_{\alpha, \beta}^{-1} g \varphi_{\alpha, \beta} .
$$

Since $\varphi_{\alpha, \beta}$ is bijective, $h$ is well defined. In particular, we have $\varphi_{\alpha, \beta} h_{\beta}=$ $\varphi_{\alpha, \beta} \varphi_{\alpha, \beta}^{-1} g \varphi_{\alpha, \beta}=g \varphi_{\alpha, \beta}=h_{\alpha} \varphi_{\alpha, \beta}$. By Proposition $3, h$ is an endomorphism on $S$. Note that $f_{\beta}=\varphi_{\alpha, \beta}^{-1} f_{\alpha} \varphi_{\alpha,(\beta) t}$ follows from Proposition 1 , since $\varphi_{\alpha, \beta}$ is bijective. Moreover, it holds $f_{\alpha}=\varphi_{\alpha, \alpha}^{-1} f_{\alpha} \varphi_{\alpha, \alpha}$ and $h_{\alpha}=\varphi_{\alpha, \alpha}^{-1} g \varphi_{\alpha, \alpha}$. Then, we obtain $f_{\alpha} h_{\alpha} f_{\alpha}=f_{\alpha} g f_{\alpha}=f_{\alpha}$ and $f_{\alpha} h_{\alpha}=f_{\alpha} g=g f_{\alpha}=h_{\alpha} f_{\alpha}$. 
On the other hand, we can calculate

$$
\begin{aligned}
f_{\beta} h_{(\beta) t} f_{(\beta) t} & =\varphi_{\alpha, \beta}^{-1} f_{\alpha} \varphi_{\alpha,(\beta) t} \varphi_{\alpha,(\beta) t}^{-1} g \varphi_{\alpha,(\beta) t} \varphi_{\alpha,(\beta) t}^{-1} f_{\alpha} \varphi_{\alpha,(\beta) t t} \\
& =\varphi_{\alpha, \beta}^{-1} f_{\alpha} g f_{\alpha} \varphi_{\alpha,(\beta) t t}=\varphi_{\alpha, \beta}^{-1} f_{\alpha} \varphi_{\alpha,(\beta) t}=f_{\beta} .
\end{aligned}
$$

To obtain $(\beta) t=(\beta) t t$, we used that $\operatorname{End}(Y)$ is idempotent, whenever $Y$ is a two-element chain. Finally, we can compute that

$$
\begin{aligned}
f_{\beta} h_{(\beta) t} & =\varphi_{\alpha, \beta}^{-1} f_{\alpha} \varphi_{\alpha,(\beta) t} \varphi_{\alpha,(\beta) t}^{-1} g \varphi_{\alpha,(\beta) t}=\varphi_{\alpha, \beta}^{-1} f_{\alpha} g \varphi_{\alpha,(\beta) t} \\
& =\varphi_{\alpha, \beta}^{-1} g f_{\alpha} \varphi_{\alpha,(\beta) t}=\varphi_{\alpha, \beta}^{-1} g \varphi_{\alpha, \beta} \varphi_{\alpha, \beta}^{-1} f_{\alpha} \varphi_{\alpha,(\beta) t}=h_{\beta} f_{\beta} .
\end{aligned}
$$

Altogether, we have shown that $f h f=f$ and $f h=h f$. Consequently, $f$ is a completely regular element of $\operatorname{End}(S)$.

Open problem. The characterization of all Clifford semigroups with completely regular endomorphism monoid is still an open problem if the structure homomorphisms $\varphi_{\alpha, \beta}$ are not surjective.

\section{References}

[1] J. Araújo, J. Konieczny, The Monoid of Holomorphic Endomorphisms of a Group and its Automorphisms, Semigroups, Acts and Categories with Applications to Graphs, Estonian Mathematical Society, 2008, pp. 7-13.

[2] Arthur H. Clifford, G. B. Preston, The algebraic theory of semigroups. Vol. I, Mathematical Surveys, No. 7, American Mathematical Society, Providence, R.I., 1961.

[3] T. Gramushnjak, P. Puusemp, A characterization of a class of 2-groups by their endomorphism semigroups. In Generalized Lie Theory in Mathematics, Physics and Beyond (Silvestrov, S. et al., eds). Springer-Verlag, Berlin, 2009, pp. 151-159.

[4] John M. Howie, An Introduction to Semigroup Theory, Acad. Press. London, 1976.

[5] A. V. Karpenko, V.M. Misyakov, On regularity of the center of the endomorphism ring of an abelian group, Journal of Mathematical Sciences, Vol. 154, 2008, pp. 304307.

[6] J. D. P. Meldrum, Regular semigroups of endomorphisms of groups, Recent Developments in the Algebraic, Analytical, and Topological Theory of Semigroups. Lecture Notes in Mathematics, Vol. 998. Springer, Berlin, Heidelberg, 2006, pp. 374-384.

[7] Mario Petrich, N. Reilly, Completely Regular Semigroups, J. Wiley, New York 1999.

[8] P. Puusemp, Idempotents of the endomorphism semigroups of groups. Acta et Comment. Univ. Tartuensis, No. 366, 1975, pp. 76-104 (in Russian).

[9] P. Puusemp, On endomorphisms of groups of order 32 with maximal subgroups $C_{4} \times C_{2} \times C_{2}$. In Proceedings of the Estonian Academy of Sciences, Vol. 63, No. 2, 2014, pp. 105-120.

[10] M. Samman, J. D. P. Meldrum, On Endomorphisms of Semilattices of Groups, Algebra Colloquium, Vol. 12, No. 01 2005, pp. 93-100.

[11] S. Worawiset, On the endomorphism monoids of Clifford Semigroups, AsianEuropean Journal of Mathematics, Vol. 11, No. 2, 2018, 1850059 (8 pages). 
Somnuek

Worawiset
CONTACT INFORMATION

Department of Mathematics, Faculty of Science, Khon Kaen University, Khon Kaen, Thailand E-Mail(s): wsomnu@kku.ac.th

Web-page(s): http://math.kku.ac.th/math2019/

Jörg Koppitz Institute of Mathematics and Informatics, Bulgarian Academy of Sciences, Acad. G. Bonchev Str. bl. 8, 1113 Sofia, Bulgaria

E-Mail(s): koppitz@math.bas.bg

Web-page $(s)$ : http:

//math.bas.bg/?page_id=2628

Received by the editors: 06.02.2020

and in final form 09.10.2020. 